\title{
A ZUPT-BASED METHOD FOR ASTRONAUT NAVIGATION ON PLANETARY SURFACE AND PERFORMANCE EVALUATION UNDER DIFFERENT LOCOMOTION PATTERNS
}

\author{
X. Sun ${ }^{\text {a, b }}$, K. Wu ${ }^{\text {a }}$, Y. Li ${ }^{\text {a, c }}$, K. Di ${ }^{\text {a, * }}$ \\ ${ }^{\text {a }}$ State Key Laboratory of Remote Sensing Science, Institute of Remote Sensing and Digital Earth, Chinese Academy of \\ Sciences, P.O.Box 9718, Datun Road, Chaoyang District, Beijing 100101, China - dikc@radi.ac.cn \\ ${ }^{\mathrm{b}}$ Computer and Information Engineering College, Henan University, Kaifeng 475001, China \\ ${ }^{c}$ Nanchang University, Nanchang 330031, China \\ Commission VI, WG IV/8
}

KEY WORDS: Planetary, Navigation, IMU, Correction, Performance

\begin{abstract}
:
In this research, we develop a Zero Velocity Update (ZUPT) based method for astronaut navigation and evaluate its performance under different locomotion patterns. In this ZUPT based dead-reckoning, the position drift of IMU is corrected using the constraint that the instantaneous velocity is zero when static state is automatically detected. A series of field experiments have been performed on terrains of different slopes and hardness in an urban park and a desert under different locomotion patterns, such as walking and hopping. The experimental results show that the positioning accuracy of the developed ZUPT based dead-reckoning is $1.5 \%-2.3 \%$ under different locomotion patterns, demonstrating the potential of ZUPT for astronaut navigation.
\end{abstract}

\section{INTRODUCTION}

Astronaut navigation system is critical to ensure an astronaut's safety and efficiency while exploring on the planetary surface in manned planetary missions (He, 2012). Accurate position information helps the astronaut find the scientific target quickly and return to re-entry module safely. Planetary surface environment not only limits the adoption of sensors (such as GPS) that are commonly used on the earth, but also causes spatial disorientation due to lack of familiar reference for vision cues of distance and size. In general, astronaut navigation on a planet surface is much more difficult than pedestrian navigation on the earth.

As Inertial Measurement Unit (IMU) can realize autonomous navigation without GPS signals and has the advantages of high data frequency, free of external influence, positioning stably within a short time, it is suitable for navigation and positioning on planetary surfaces. A small-sized micro-electromechanical system (MEMS) is an optimal choice for astronaut navigation. By integrating measurements from IMU, position and attitude information can be obtained at a high frequency. However, this dead reckoning method suffers from severe drift and is bound to accumulate with time and cannot be directly used for navigation applications. Without external aiding measurements, positioning error can sharply increase within seconds. In the field of pedestrian navigation, Zero Velocity Update (ZUPT) (Elwell, 1999; Foxlin, 2005) is a commonly used method for restraining error accumulation of IMU by correcting error drift using the constraint that the instantaneous velocity turns zero when the person stops walking. Based on this principle, most IMUs used in personal navigation are mounted on the foot (Nilsson et al., 2012).

\footnotetext{
* Corresponding author.
}

The most important part of the ZUPT algorithm is the detection of stationary state. There are several methods for stationary state detection such as using knowledge of the human walking pattern or the sensor's stationary data (Fischer et al., 2013; Park et al., 2010). In astronaut navigation, all locomotion patterns, such as walking and hopping, have the property that the feet repeat periodical motion mode containing regular reoccurring stationary periods when they touch the ground. When foodmounted MEMS returns to stationary state, there is usually a short stay for about tenths of a second, which is called zero velocity intervals. In a ZUPT algorithm, zero velocity intervals are detected first and then velocities are set to zero accordingly. Due to the existence of noise, zero velocity intervals are usually hard to detect, which leads to a wrong judge of zero velocity.

In this paper, a ZUPT method, including zero velocity detection and update, is implemented for simulative astronaut navigation on planetary surface. A number of field experiments are performed for testing the applicability of the method, positioning accuracy is evaluated under different locomotion patterns.

\section{METHOD}

\subsection{Zero Velocity Detection}

Mathematically, detection problem is a binary hypothesis testing problem. For explaining the test statistics of zero velocity detection, we first define three conditions for judging stationary state.

(a) In stationary state, no external force except gravity acts on the MEMS, so the specific force $\left\|f_{n}\right\|$ at the time instant $n$ should equal to the local gravity value theoretically as shown in Equation (1). 


$$
\left\|f_{n}\right\|=\sqrt{f_{\mathrm{x}}^{2}+f_{\mathrm{y}}^{2}+f_{\mathrm{z}}^{2}}
$$

where $f_{\mathrm{x}}, f_{\mathrm{y}}$ and $f_{\mathrm{z}}$ are the components of the specific force in $\mathrm{X}-$, $\mathrm{Y}$ - and Z- axis directions at the time $n$. Thus, a constraint is determined that the specific force $\left\|f_{n}\right\|$ at a stationary instant should be in a certain range around gravity.

(b) If there is a zero velocity interval in the detection window, the variance of the specific forces in the window should be less than a pre-defined threshold.

$$
\sigma^{2}(f)=\frac{1}{\mathrm{~W}} \sum_{k=n}^{n+\mathrm{W}-1}\left\|f_{k}-\bar{f}_{k}\right\|^{2}
$$

where $\bar{f}_{k}$ is the mean value of the specific forces.

(c) Angular rate is also considered in detecting stationary state. Similar to Equation (1), angular rate $\omega_{n}$ at the time instant $n$ is defined as

$$
\left\|\omega_{n}\right\|=\sqrt{\omega_{\mathrm{x}}^{2}+\omega_{\mathrm{y}}^{2}+\omega_{\mathrm{z}}^{2}}
$$

where $\omega_{\mathrm{x}}, \omega_{\mathrm{y}}$ and $\omega_{\mathrm{z}}$ are the components of the angular rate in $\mathrm{X}-, \mathrm{Y}-$ and $\mathrm{Z}$ - axis directions at the time $n$. In stationary state, angular rate $\omega_{n}$ is theoretically equal to zero as well.

Combining the three conditions above, a test statistics of zero velocity detection is given (Skog et al., 2010):

$$
T=\frac{1}{\mathrm{~W}} \sum_{k=n}^{n+\mathrm{W}-1} \frac{1}{\sigma_{f}^{2}}\left\|f_{k}-g \frac{\bar{f}_{k}}{\left\|\bar{f}_{k}\right\|}\right\|^{2}+\frac{1}{\sigma_{\omega}^{2}}\left\|\omega_{k}\right\|^{2}
$$

where $g$ is the gravity value, $\sigma_{f}$ and $\sigma_{\omega}$ are measurement errors (standard deviations), $\gamma$ is the pre-defined zero velocity detection threshold.

\subsection{Zero Velocity Update}

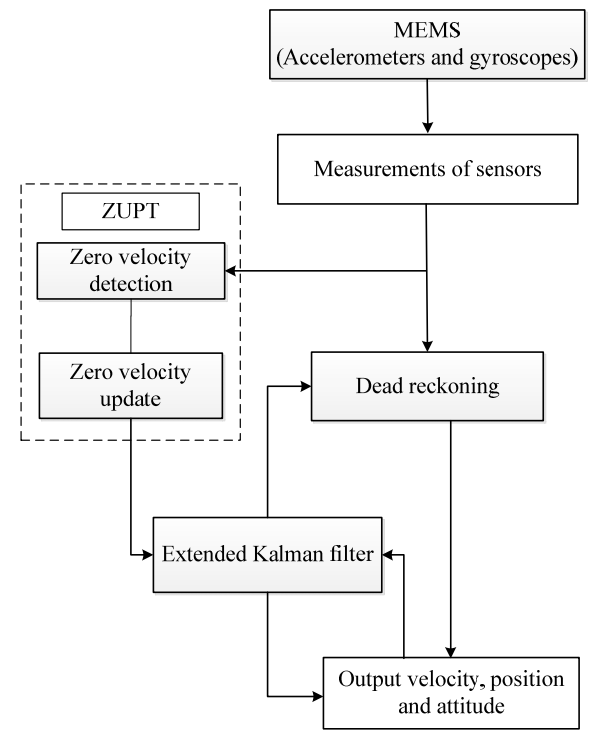

Figure 1. A diagram of zero-velocity-constrained deadreckoning with MEMS
The diagram of zero-velocity-constrained dead-reckoning with MEMS is shown in

Figure 1. An extended Kalman filter method is adopted in this paper to estimate the velocity, correct the position and calculate the orientation using the measurements of the accelerometers and gyroscopes of the MEMS.

\section{FIELD EXPERIMENTS}

In order to validate the developed ZUPT based dead-reckoning (ZUPT-DR) algorithm and evaluate the navigation performance under different locomotion patterns, we established an experimental facility and performed field tests along different paths. As shown in Figure 2, the experimental facility includes an Xsens MTi MEMS mounted on one foot and a Mini PC in a backpack.

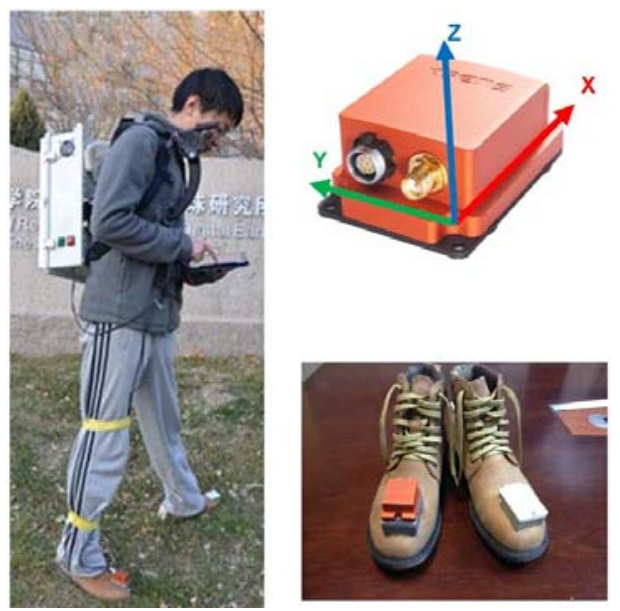

Figure 2. The experimental facility

Table 1 lists the technical parameters of the accelerometers and gyroscopes. The MEMS has been calibrated and has an output frequency of 200 samples per second. In our experiments, the MEMS is mounted on the right foot, with $\mathrm{X}$-axis pointing forward, Y-axis pointing to the left (the inside of the right foot), $\mathrm{Z}$-axis is perpendicular to the $\mathrm{X}-\mathrm{Y}$ plane to complete a righthand coordinate system (see Figure 2).

Table 1. Technical parameters of the MTi MEMS

\begin{tabular}{|l|l|l|}
\hline & Accelerometers & Gyroscopes \\
\hline Full Range & $\pm 50 \mathrm{~m} / \mathrm{s}^{2}$ & $\pm 450^{\circ} / \mathrm{s}$ \\
\hline Non-linearity & $0.03 \% \mathrm{FS}$ & $0.01 \% \mathrm{FS}$ \\
\hline Noise density & $80 \mu \mathrm{g} / \sqrt{\mathrm{Hz}}$ & $0.01 \% / \mathrm{s} / \sqrt{\mathrm{Hz}}$ \\
\hline Bias repeatability & $0.03 \mathrm{~m} / \mathrm{s}^{2}$ & $0.2^{\circ} / \mathrm{s}$ \\
\hline Bandwidth & $375 \mathrm{~Hz}$ & $415 \mathrm{~Hz}$ \\
\hline
\end{tabular}

Field experiments were performed in a park in Beijing and a small desert in Hebei Province for simulating the planetary surface environment. Due to the absence of high precision GPS measurement, distances of all the paths in the experiments were measured by a tape or a distance measuring wheel and used as true values.

Four groups of experiments including walking, hopping, uphill and downhill patterns with 50 to 100 meters paths were designed for evaluating the effectiveness of the ZUPT-DR algorithm. 
In the first group of experiments, the experimenter walked straight for 78 seconds and 156 seconds, with a total distance of 50 meters and 100 meters respectively. Figures 3 and 4 show the paths recovered by the ZUPT-DR algorithm. Positioning errors calculated by ZUPT-DR are about 0.91 meter and 1.64 meters, thus a relative error of less than $2 \%$ is achieved.

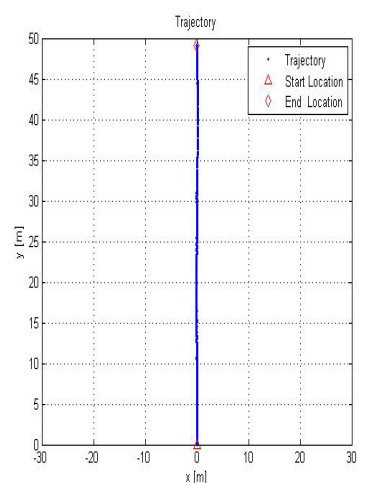

Figure 3. Straight line path of 50 meters

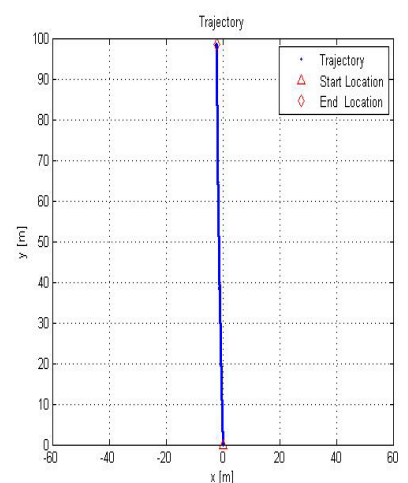

Figure 4. Straight line path of 100 meters
In the second group of experiments, the experimenter walked along a closed rectangular path with total distances of 80 meters and 100 meters and the estimated accuracies are also less than $2 \%$. Figures 5 and 6 show the recovered paths by the ZUPT-DR.

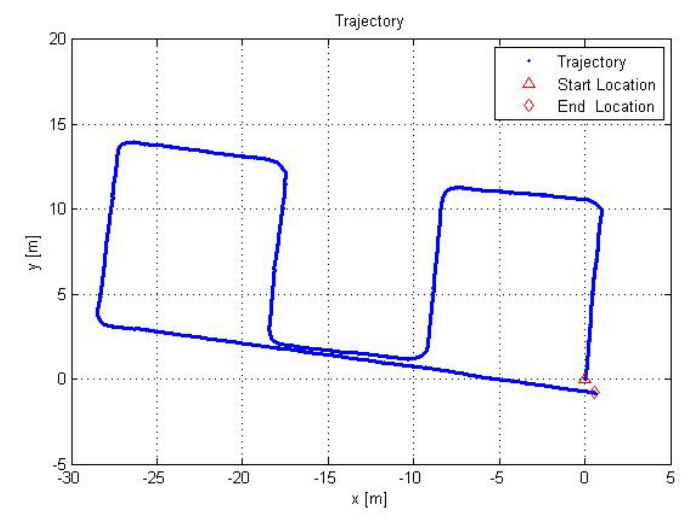

Figure 5. Closed path of 100 meters

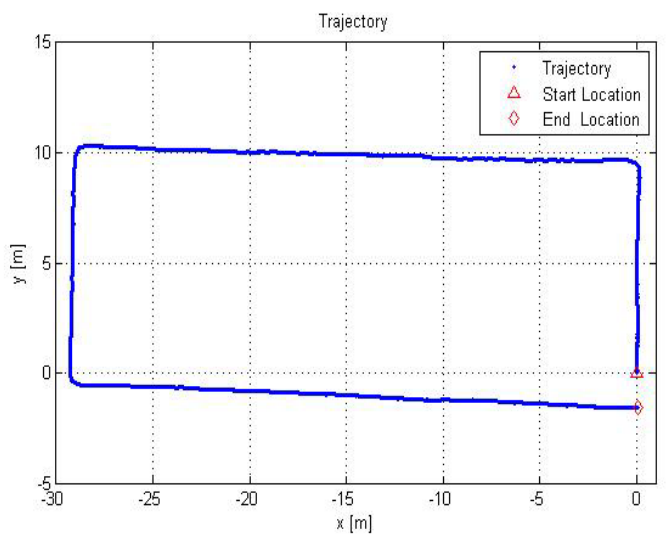

Figure 6. Closed path of 80 meters

The above experiments were carried out in the park with hard surface. The following experiments were carried out in the desert. Figure 7 shows a scene of the experiments in the desert. In the third group of experiments, the performances of uphill and downhill walking on surfaces of different levels of hardness and slope were tested. The recovered paths are shown in Figures 8 and 9 .

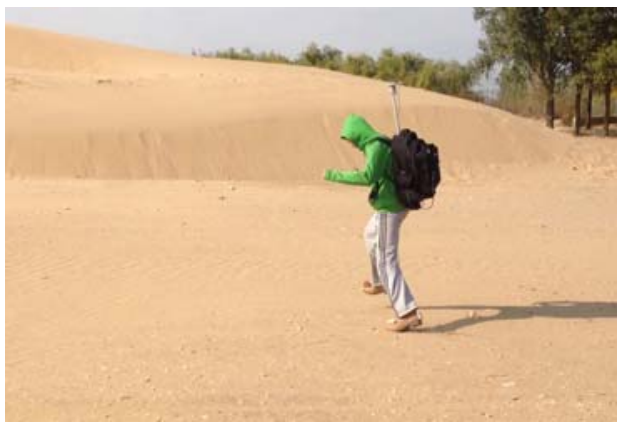

Figure 7. Field experiments in a desert

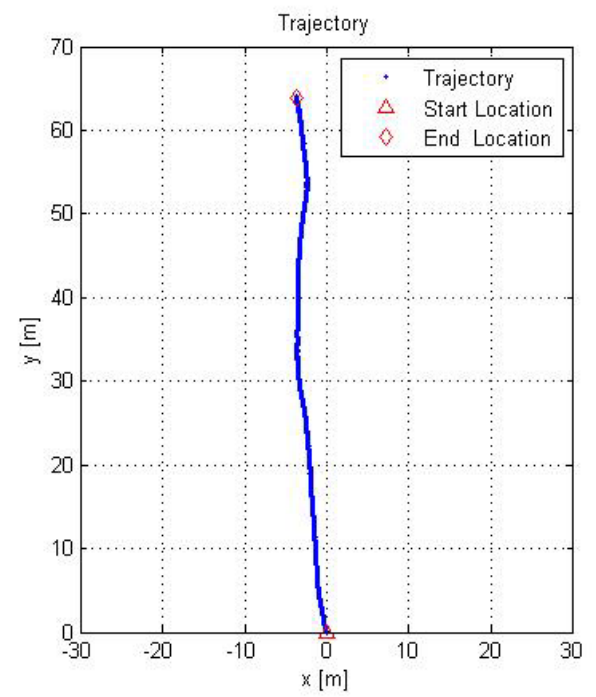

Figure 8. Uphill walk path of 66.46 meters

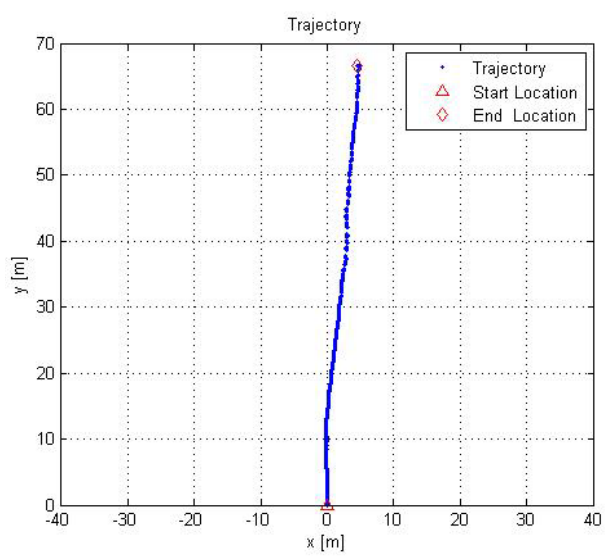

Figure 9. Downhill walk path of 68.04 meters

In the fourth group of experiments, the performances of hopping along a straight line of 50 meters and 100 meters were tested. The recovered paths are shown in Figures 10 and 11. We also tried to test the hopping pattern in very soft sand; data acquisition was not successful because hopping on such surface was too difficult at $1 \mathrm{~g}$ gravity on the earth. 


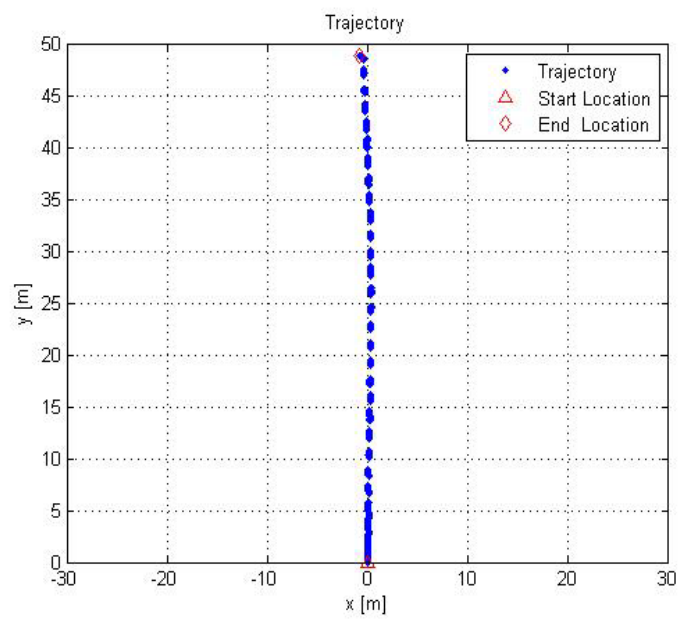

Figure 10. Hopping path of 50 meters

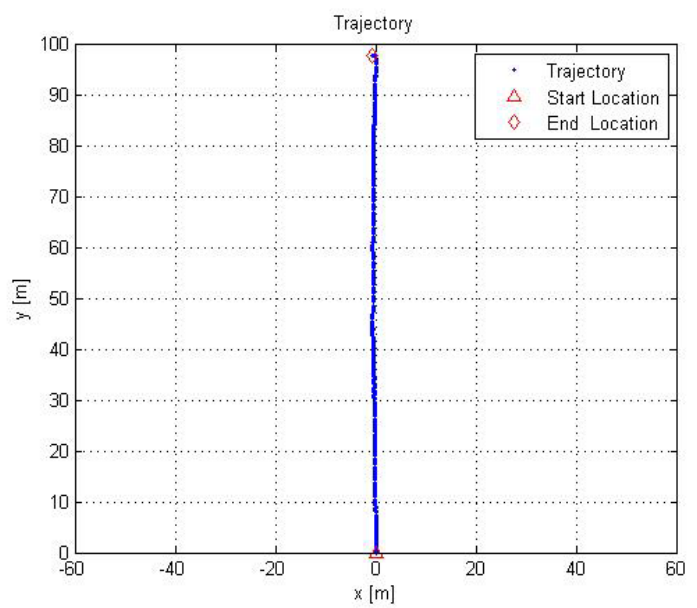

Figure 11. Hopping path of 100 meters

All the experimental results are summarized in Table 2. The reference ("true") distances of uphill and downhill walks were measured by a distance measuring wheel, all the other references distances were measured by a tape. It can be seen that the positioning accuracy of walking under different conditions is stable and below $2 \%$ of the total distance, and the positioning accuracy for the hopping pattern is slightly lower than that of walking pattern and is below $2.3 \%$.

Table 2. The positioning accuracies in the experiments

\begin{tabular}{|c|c|c|c|}
\hline \multirow{2}{*}{$\begin{array}{c}\text { Locomotion } \\
\text { patterns }\end{array}$} & $\begin{array}{c}\text { Reference } \\
\text { Distance } \\
(\mathrm{m})\end{array}$ & $\begin{array}{c}\text { Distance from } \\
\text { ZUPT-DR } \\
(\mathrm{m})\end{array}$ & $\begin{array}{c}\text { Relative } \\
\text { Error }\end{array}$ \\
\hline $\begin{array}{c}\text { Straight } \\
\text { line walk }\end{array}$ & 50 & 49.09 & $1.82 \%$ \\
\hline \multirow{2}{*}{$\begin{array}{c}\text { Closed } \\
\text { path walk }\end{array}$} & 100 & 98.36 & $1.64 \%$ \\
\cline { 2 - 4 } Uphill walk & 66.46 & 98.14 & $1.86 \%$ \\
\hline $\begin{array}{c}\text { Downhill } \\
\text { walk }\end{array}$ & 68.04 & 78.42 & $1.98 \%$ \\
\hline \multirow{2}{*}{ Hopping } & 50 & 66.81 & $1.91 \%$ \\
\cline { 2 - 4 } & 100 & 98.9 & $2.2 \%$ \\
\hline
\end{tabular}

\section{CONCLUSIONS}

In this research, a ZUPT-based dead-reckoning method was implemented for simulative astronaut navigation on planetary surface and the performances of the algorithm have been evaluated under different locomotion patterns along different paths. A series of field experiments were performed and the results show that the positioning accuracy of the developed ZUPT-DR is $1.5 \%-2.3 \%$ under different locomotion patterns, proving that ZUPT is effective to reduce positioning error. It should be noted that ZUPT only improves positioning accuracy with the orientation results unimproved. Recently, our team has developed a navigation method combining monocular visual odometry and laser distance meter for astronaut navigation $(\mathrm{Wu}$ et al., 2014). In the future, we will integrate the ZUPT-DR into the visual odometry system to enhance the overall astronaut navigation capability. In such an integrated system, the visual sensor can provide attitude information to reduce the orientation errors of the MEMS, while the ZUPT-DR can compensate the visual odometry when lacking of visual landmarks.

\section{ACKNOWLEGEMENTS}

The work described in this paper is supported by National Natural Science Foundation of China (41171355).

\section{REFERENCES}

Elwell, J., 1999. Inertial navigation for the urban warrior. In: AeroSense'99 International Society for Optics and Photonics, Raja Suresh, Orlando, FL, USA, pp. 196-204.

Fischer, C., Talkad Sukumar, P. and Hazas, M., 2013. Tutorial: implementation of a pedestrian tracker using foot-mounted inertial sensors. IEEE Pervasive Computing, 12(2), pp. 17-27.

Foxlin, E., 2005. Pedestrian tracking with shoe-mounted inertial sensors. Computer Graphics and Applications, IEEE, 25(6), pp. $38-46$.

He, S., 2012. Integration of multiple sensors for astronaut navigation on the lunar surface. Ph.D. dissertation, The Ohio State University.

Nilsson, J.-O., Skog, I., Handel, P. and Hari, K., 2012. Footmounted INS for everybody - an open-source embedded implementation. In: Position Location and Navigation Symposium, Myrtle Beach, SC, USA, pp. 140-145.

Park, S. K., and Suh, Y. S., 2010. A zero velocity detection algorithm using inertial sensors for pedestrian navigation systems. Sensors, 10(10), pp. 9163-9178.

Skog, I., Nilsson, J.-O., and Handel, P., 2010. Evaluation of zero-velocity detectors for foot-mounted inertial navigation systems. In: 2010 International Conference on Indoor Positioning and Indoor Navigation, Zurich, Switzerland, pp. 16.

Wu, K., Di, K., Sun, X., Wan W., and Liu, Z., 2014. Enhanced Monocular Visual Odometry Integrated with Laser Distance Meter for Astronaut Navigation. Sensors,14, pp. 4981-5003 